\title{
Ethylparaben induces subconjunctival fibrosis via the Wnt/及-catenin signaling pathway
}

\author{
FENGGE LIU, XIANGFENG KONG and HUI KONG \\ Department of Ophthalmology, Zoucheng People's Hospital, Zoucheng, Shandong 273500, P.R. China
}

Received April 12, 2018; Accepted November 1, 2018

DOI: $10.3892 /$ etm.2021.9726

\begin{abstract}
The aim of the present study was to explore the etiology of subconjunctival fibrosis (SCF) induced by ethylparaben, the most prevalent preservative in Chinese eye drops. Ethylparaben was administered to the left eyes of male Sprague-Dawley rats in the experimental group twice daily for 1 month, whereas the control group received PBS. Experimental group rats displayed a mild promotion in density of fibroblasts and a tighter deposition of collagen in the bulbar subepithelial connective tissue compared with the control group. Furthermore, the present findings revealed that extracellular matrix expression was promoted in murine bulbar conjunctival tissues in the experimental group. In primary conjunctival fibroblasts, expression of ECM triggered by ethylparaben was suppressed by XAV-939. Furthermore, stimulation of the $\mathrm{Wnt} / \beta$-catenin axis triggered by ethylparaben was impaired by XAV-939. In conclusion, SCF triggered by ethylparaben results from extra ECM generation of conjunctival fibroblasts via the $\mathrm{Wnt} / \beta$-catenin axis.
\end{abstract}

\section{Introduction}

Ethylparaben is a widely used preservative in Chinese eye drops $(1,2)$. Subconjunctival fibrosis (SCF) leads to failure of glaucoma filtration surgery, inducing conjunctival scarring during wound healing (3-5). Nevertheless, the action of ethylparaben in SCF remains to be elucidated.

The Wnt/ $\beta$-catenin axis participates in development and in tissue homeostasis, and is divided into canonical and non-canonical signaling (6,7). Abnormal stimulation of the canonical Wnt pathway stimulates various pathological states including malignancies (8). The transcription factor complex, consisting of $\beta$-catenin, $\mathrm{T}$ cell factor and lymphoid enhancer factor, is widely recognized as the main modulator of the

Correspondence to: Dr Xiangfeng Kong, Department of Ophthalmology, Zoucheng People's Hospital, 59 Qianquan Road, Zoucheng, Shandong 273500, P.R. China

E-mail: kongxf63@163.com

Key words: ethylparaben, subconjunctival fibrosis, extracellular matrix, Wnt/ $\beta$-catenin, XAV-939
Wnt/ $\beta$-catenin axis $(9,10)$. When the Wnt ligand is not activated, a destroying complex consisting of the tumor suppressor adenomatous polyposis coli, axin and glycogen synthase kinase-3 (GSK3 $\beta$ ) anchors cytoplasmic $\beta$-catenin (11-13). GSK $3 \beta$ and casein kinase 1 participate in the phosphorylation of cytoplasmic $\beta$-catenin, bringing about ubiquitination and degeneration of proteasome $(14,15)$. When the Wnt ligand is activated, it links with the Frizzled receptor and its co-receptor, low-density lipoprotein, by a receptor-related protein $5 / 6$ on the surface of the cell. This propels dishevelled to attract GSK $3 \beta$ and Axin, consequently separating $\beta$-catenin from the destroying complex $(16,17)$. Degeneration and phosphorylation of $\beta$-catenin is subsequently suppressed prior to aggregation of cytoplasmic $\beta$-catenin and nuclear translocation (18). This association induces the expression of target genes in the Wnt $/ \beta$-catenin axis. Previous studies have demonstrated that the Wnt/ $\beta$-catenin axis participates in corneal fibrosis $(19,20)$. Nevertheless, the current understanding of the influence of stimulation of the Wnt/ $\beta$-catenin axis on SCF triggered by ethylparaben is insufficient.

The present study explored the etiology of SCF triggered by ethylparaben through examination of the concentration of the extracellular matrix (ECM) and agents linked with the $\mathrm{Wnt} / \beta$-catenin axis in vivo and in vitro. It was discovered that deposition of collagen in murine conjunctival subepithelium was triggered by ethylparaben supplemented for one month, and that the $\mathrm{Wnt} / \beta$-catenin axis was crucial to this reaction.

\section{Materials and methods}

Materials. Dulbecco's Modified Eagle Medium (DMEM) and fetal bovine serum (FBS) were purchased from Invitrogen; Thermo Fisher Scientific, Inc. (Waltham, MA, USA). Ethylparaben was provided by Sigma-Aldrich; Merck KGaA (Darmstadt, Germany). XAV-939 was obtained from Santa Cruz Biotechnology, Inc. (Dallas, TX, USA) and used at $1 \mu \mathrm{M}$.

Animals and ethylparaben treatment. Experiments involving animal subjects in the present study were conducted in accordance with the internationally accepted principles for laboratory animal use and care. The present study was approved by the Committee on the Ethics of Animal Experiments of Zoucheng People's Hospital (Zoucheng, China). Sprague-Dawley rats (age, 6 weeks; weight, $215 \pm 30 \mathrm{~g}$ ) were obtained from the Beijing Vital River Laboratory 
Animal Technology Co., Ltd. (Beijing, China) and used in the present study. Rats were housed in microisolator cages in a room illuminated from 0700-1900 h (12-h light/dark cycle; humidity, $55 \pm 5 \% ; 25 \pm 2^{\circ} \mathrm{C}$ ), with ad libitum access to water and chow. Animal procedures were carried out in conformity to guidelines of the ARVO Statement for the Use of Animals in Ophthalmic and Vision Research (21). Each rat was verified for ocular surface illness prior to experimentation. A total of 18 rats were randomized into 3 groups ( $n=6 /$ group). In the first group, $0.01 \%$ ethylparaben in PBS was dropped locally into the left eye (twice daily, $10 \mu \mathrm{l}$ each). In the second group (control group), PBS was supplemented the same way (twice daily, $10 \mu l$ each). All rats were executed 1 month following supplementation. Bulbar conjunctival tissues (BCTs) were excised to separate RNA and protein. Rats in the third group received no treatment prior to sacrifice and their left eyes were enucleated under aseptic circumstances for separation of bulbar subconjunctival tissues (BSTs).

Cultivation of primary conjunctival fibroblasts (CFs). Primary CFs were separated from BSTs and cultivated. BSTs separated under aseptic circumstances were washed thrice with PBS. BSTs were subsequently processed into $2 \times 2-\mathrm{mm}$ explants, which were planted in cultivating plates at a density of $5 \times 10^{3}$ cells/well filled with DMEM and 10\% FBS and were incubated in an environment with $5 \% \mathrm{CO}_{2}, 95 \%$ humidity, and $37^{\circ} \mathrm{C}$. The medium was replaced every three days. CFs were subcultivated with trypsin for procedures between passages five and seven.

In order to examine the influence of low levels of ethylparaben on $\mathrm{CF}$ survival and to explore the optimal ethylparaben level for examining the etiology of ECM gene expression of CFs activated by ethylparaben, $\mathrm{CFs}$ were planted in plates with 96 wells $\left(3 \times 10^{3}\right.$ cells/well) and received media with either ethylparaben $(0.000005 \%, 0.00001 \%, 0.00003 \%, 0.00005 \%$, $0.0001 \%, 0.001 \%)$ or PBS at $37^{\circ} \mathrm{C}$.

Analysis of $\beta$-catenin nuclear translocation. CFs were treated with ethylparaben for $4 \mathrm{~h}$. Nuclear fractionation was used to analyze $\beta$-catenin nuclear translocation. Briefly, nuclear extracts were isolated from CFs treated in $75-\mathrm{cm}^{2}$ flasks using the NEPER Nuclear/Cytoplasmic Extraction Kit (Thermo Fisher Scientific, Inc.) according to the manufacturer's instructions, and analyzed by $\beta$-catenin WB analysis.

Western blotting $(W B)$. Western blotting was performed as previously detailed (22-24). The total proteins were extracted from $\mathrm{BCTs}$, and the $\mathrm{CFs}$ were extracted using a cold radioimmunoprecipitation assay buffer that contains a proteinase inhibitor cocktail (Roche Diagnostics, Basel, Switzerland). Protein concentration was determined using a Piece BCA Protein Assay kit (Thermo Fisher Scientific, Inc.). Equivalent quantities of proteins $(50 \mu \mathrm{g})$ harvested from CFs and conjunctival tissues were subjected to electrophoresis with $10 \%$ SDS-PAGE. Proteins were transferred onto polyviylidene difluoride membranes. Subsequently, the membranes were blocked at room temperature for $1 \mathrm{~h}$ using $5 \%$ not-fat milk in tris-buffered saline and incubated with primary antibodies against: $\alpha$-smooth muscle actin (SMA; cat. no. ab5694), collagen 1 (cat. no. ab138492), fibronectin
A

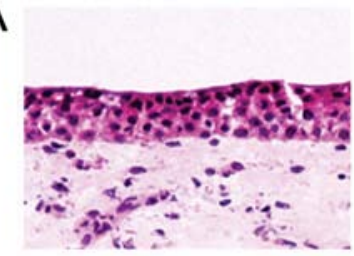

PBS
B

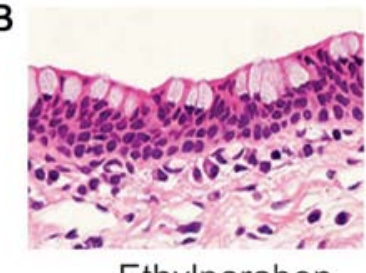

Ethylparaben

Figure 1. Histological staining of rat conjunctival tissues. Subepithelial areas of the BC in the (A) PBS- and (B) ethylparaben-treated groups were detected via hematoxylin and eosin staining (magnification, $\mathrm{x} 20$ ). The density of fibroblasts was markedly promoted and the deposition of collagen was tighter in the subepithelium of BCs in the experimental group than the control group. $\mathrm{BC}$, bulbar conjunctiva.

(FN)-1 (cat. no. ab2413; all Abcam, Cambridge, UK), $\beta$-actin (cat. no. 3700), collagen type 1 (Col1) $\alpha 1$ (cat. no. 84336), phosphorylated (p)- $\beta$-catenin (cat. no. 4176), $\beta$-catenin (cat. no. 8480), Lamin A/C (cat. no. 4777; all Cell Signaling Technology, Danvers, MA, USA) at 1:1,000 dilution and $4^{\circ} \mathrm{C}$ overnight. The membranes were washed and then incubated with horseradish peroxidase-conjugated secondary antibodies (1:10,000; cat. no. ab6721; Abcam,) for $1 \mathrm{~h}$ at room temperature. Finally, an enhanced chemiluminescence ECL Detection kit (Thermo Fisher Scientific, Inc.) was utilized to evaluate the bands, and a transilluminator (Bio-Rad Laboratories, Inc., Hercules, CA, USA) was used to examine the intensity of the image.

Cell survival assessment. A cell counting kit-8 (CCK-8; Sigma-Aldrich; Merck KGaA) was used to assess survival. The media was replaced with fresh media with CCK-8 solution. Following a 2-h period of incubation at $37^{\circ} \mathrm{C}$, the microplate reader was used to spectrophotometrically assess absorbance at $450 \mathrm{~nm}$.

Reverse transcription-quantitative polymerase chain reaction ( $R T-q P C R)$. TRIzol reagent (Invitrogen; Thermo Fisher Scientific, Inc.) was used to isolate total RNA from BCTs, which then underwent reverse transcription to cDNA using a First Stand cDNA synthesis kit (Sigma-Aldrich; Merck KGaA). qPCR was performed in triplicate using SYBR Premix Ex Taq (Takara Biotechnology Co., Ltd., Dalian, China) and SsoFast ${ }^{\mathrm{TM}}$ Probes Supermix (Bio-Rad Laboratories, Inc.). The following thermocycling conditions were used for the RT-qPCR: Initial denaturation at $95^{\circ} \mathrm{C}$ for $1 \mathrm{~min} ; 38$ cycles for $95^{\circ} \mathrm{C}$ for $1 \mathrm{~min}, 58^{\circ} \mathrm{C}$ for $1 \mathrm{~min}, 72^{\circ} \mathrm{C}$ for $1 \mathrm{~min}$; and a terminal extension at $72^{\circ} \mathrm{C}$ for $10 \mathrm{~min}$. The outcome of RT-qPCR was evaluated using the $2^{-\Delta \Delta C q}$ approach (25) and was normalized in conformity with $\beta$-actin. The primers used were as follows: Coll $\alpha 1$, forward 5'-CCTCCCAGAACATTACATA-3' and reverse 5'-GACTGT CTTGCTCCATTCACCA-3'; FN-1, forward 5'-GACTCG TTTGACTTTGACTTCACCAC-3' and reverse 5'-GCTGAG ACCCAGGAGACCAC-3'; collagen 1, forward 5'-TACAGC ACGCTTGTGGATG-3' and reverse 5'-CAGATTGGGATG GAGGGAGTT-3'; $\alpha$-SMA, forward 5'-GAGGCACCACTG AACCCTAA-3' and reverse 5'-CATCTCCAGAGTCCAGCA CA-3'; $\beta$-catenin, forward 5'-GCTGACCTGATGGAGTTG GA-3' and reverse 5'-GCTACTTGCTCTTGCGTGAA-3'; 

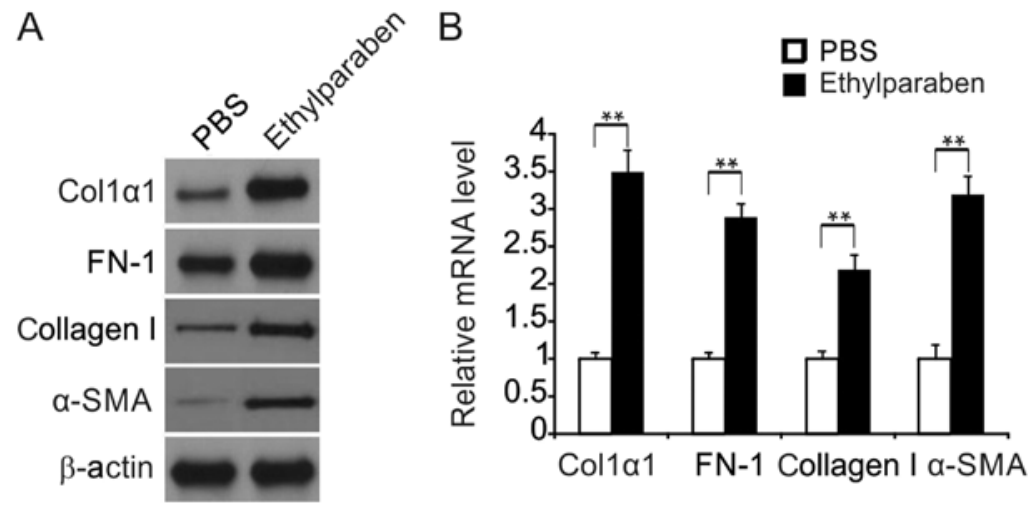

Figure 2. Expression of extracellular matrix genes. (A) The protein expression of FN-1, Col1 $\alpha 1$, collagen I and $\alpha$-SMA was determined by western blotting (B) The mRNA expression levels of FN-1, Coll $\alpha 1$, collagen I and $\alpha$-SMA were determined by reverse transcription-quantitative polymerase chain reaction. Expression and translation of FN-1, Coll $\alpha 1$, collagen I and $\alpha$-SMA were promoted with ethylparaben treatment. ${ }^{* *} \mathrm{P}<0.01$. FN, fibronectin; Col1, collagen type 1; SMA, smooth muscle actin.

and $\beta$-actin, forward 5'-GACGTTGACATCCGTAAAGAC C-3' and reverse 5'-CTAGGAGCCAGGGCAGTAATCT-3'.

Hematoxylin and eosin $(H \& E)$ staining. $\mathrm{H} \& \mathrm{E}$ staining was carried out in $5-\mu \mathrm{m}$-thick paraffin-embedded sections of murine eye specimens, which were fixed with $4 \%$ paraformaldehyde at $25^{\circ} \mathrm{C}$ for $30 \mathrm{~min}$. The slices underwent deparaffinization (xylene, $3 \mathrm{~min}$ ) and rehydration $(100 \%$ ethanol, $3 \mathrm{~min}$; 95\% ethanol, $3 \mathrm{~min}$; 70\% ethanol, $3 \mathrm{~min}$; $50 \%$ ethanol, $3 \mathrm{~min}$ ) prior to $\mathrm{H} \& \mathrm{E}$ staining $(1 \mathrm{~min} / \mathrm{each})$ for histopathological observation at $25^{\circ} \mathrm{C}$. A Nikon Diaphot 200 inverted microscope (Nikon Corporation, Tokyo, Japan) was used to evaluate images.

Statistical analysis. Data are presented as the mean \pm standard deviation. Student's t-test was used to identify the significance of differences between two groups, and one-way analysis of variance followed by Tukey's test was used for analysis of multiple groups. $\mathrm{P}<0.05$ was considered to indicate a statistically significant difference. GraphPad Prism IV software (GraphPad Software, Inc., La Jolla, CA, USA) was used for analyses.

\section{Results}

Chronic supplement of $0.01 \%$ ethylparaben induces murine bulbar SCF. In order to examine the influence of chronic supplementation of $0.01 \%$ ethylparaben on SCF in murine bulbar conjunctivas (BCs), the present study investigated slices of murine eye specimens that underwent $H \& E$ staining. $\mathrm{H} \& \mathrm{E}$ staining demonstrated that the density of fibroblasts was mildly promoted and the deposition of collagen was tighter in the subepithelium of BCs in the experimental group than the control group (Fig. 1). These findings suggested that chronic supplementation of ethylparaben triggered bulbar subconjunctival fibrosis (BSF).

Chronic supplementation of $0.01 \%$ ethylparaben enhances the expression of ECM in BCT. In order to verify that BSF triggered by chronic supplementation of $0.01 \%$ ethylparaben resulted from increased collagen production and other ECM material in BCTs, FN-1 and Col1 $\alpha 1$ expression was evaluated in murine
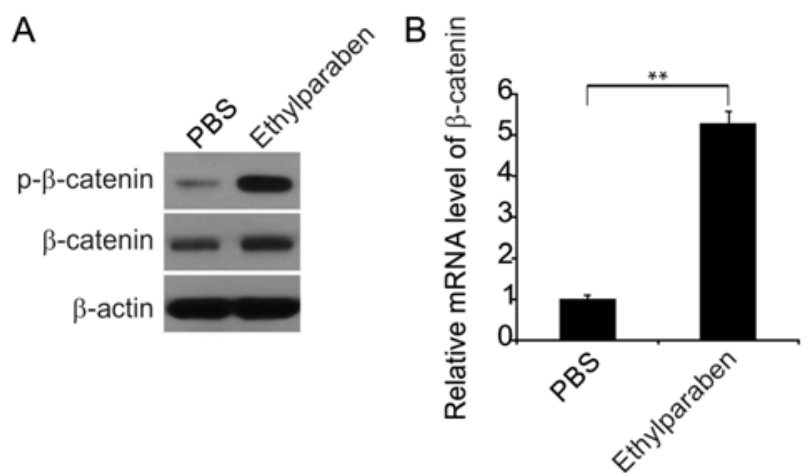

Figure 3. Expression of $\beta$-catenin. (A) Western blotting of the total and $\mathrm{p}-\beta$-catenin levels in the PBS and ethylparaben-treated groups. Phosphorylated and total $\beta$-catenin levels were markedly upregulated in the ethylparaben-treated group (B) Reverse transcription-quantitative polymerase chain reaction of the expression of $\beta$-catenin in the PBS- and ethylparaben-treated groups. $\beta$-catenin transcription was noticeably promoted in the experimental group. Data are representative examples of three independent experiments. *" $\mathrm{P}<0.01$. $\mathrm{P}$, phosphorylated.

BCTs using WB. Expression of FN-1, Col1 $\alpha 1$, collagen I and $\alpha$-SMA were markedly promoted in the experimental group compared with the control group (Fig. 2A). The mRNA level of these genes was also promoted in the experimental group according to RT-qPCR (Fig. 2B).

Chronic supplementation of $0.01 \%$ ethylparaben activates the expression of agents linked with the Wnt/ $\beta$-catenin axis in BCTs. It has been demonstrated previously that the Wnt/ $\beta$-catenin axis participates in fibrosis $(26,27)$. To explore the stimulation of the Wnt/ $\beta$-catenin axis by chronic treatment with $0.01 \%$ ethylparaben, the expression of agents linked with the $\beta$-catenin pathway was explored in murine BCTs using WB. It was observed that both $\mathrm{p}$ - and total $\beta$-catenin levels were markedly upregulated in the ethylparaben-treated group compared with the control group (Fig. 3A), and that the $\beta$-catenin transcription was noticeably promoted in the experimental group according to RT-qPCR (Fig. 3B). These findings suggested that chronic supplementation of $0.01 \%$ ethylparaben was able to trigger stimulation of the Wnt/ $/$-catenin axis, ECM overproduction and bringing about BSF. 

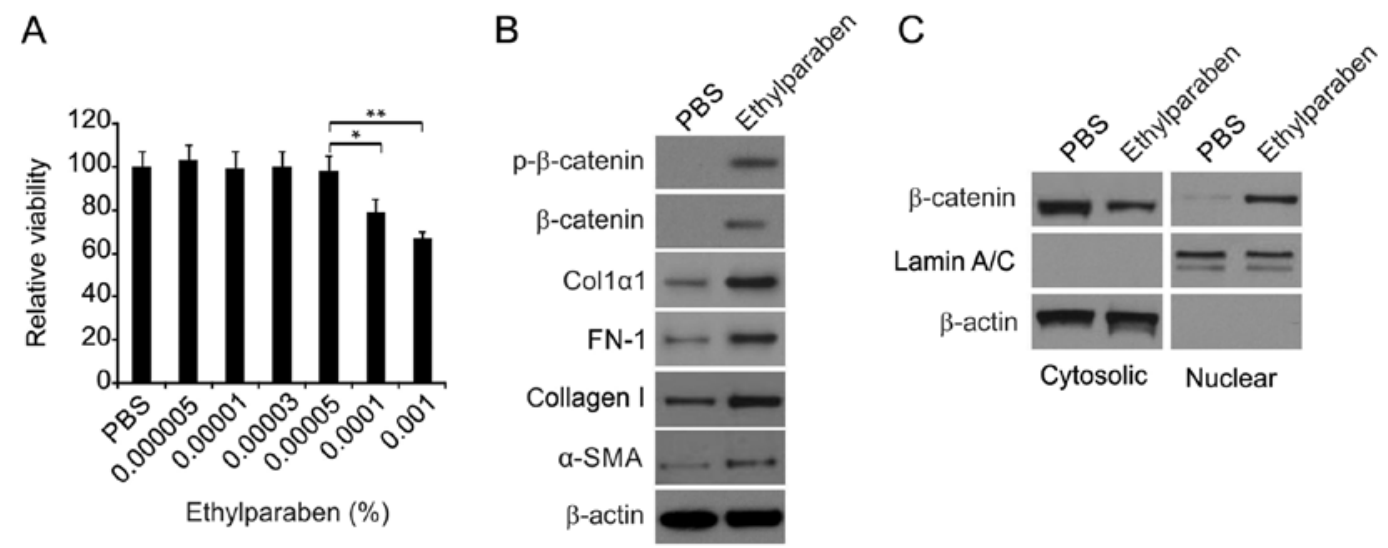

Figure 4. Effects of ethylparaben on primary CFs. (A) The effect of ethylparaben on CFs was determined via cell counting kit- 8 assay. The data are representative examples of three independent experiments. "P<0.05 and ${ }^{* *} \mathrm{P}<0.01$. (B) Western blotting assay of expression of FN-1, Coll $\alpha 1$, collagen I and $\alpha$-SMA, total and p- $\beta$-catenin in the PBS and ethylparaben-treated groups. (C) Nuclear fractions were isolated from CFs and analyzed for $\beta$-catenin by western blotting. The expression of FN-1, Coll $\alpha 1$, collagen I, $\alpha$-SMA, total and phosphorylated $\beta$-catenin was markedly promoted at $24 \mathrm{~h}$ following supplementation of CFs with 0.00005\% ethylparaben. CFs, conjunctival fibroblasts; FN, fibronectin; Col1, collagen type 1; SMA, smooth muscle actin; p, phosphorylated.

Influence of ethylparaben on the expression of ECM and agents is associated with the Wnt/ $\beta$-catenin axis in primary $C F s$. In order to reveal the etiology of SCF triggered by $0.01 \%$ ethylparaben, the influence of ethylparaben on the expression of ECM and agents linked with the Wnt/ $\beta$-catenin axis in cultured murine primary $\mathrm{CFs}$ was explored.

In order to explore the influence of ethylparaben at low levels on CF survival, and to elucidate the optimal level of ethylparaben to reveal the etiology of the expression of ECM and agents linked with the $\mathrm{Wnt} / \beta$-catenin axis, $\mathrm{CFs}$ were supplemented with media containing either ethylparaben or PBS for $24 \mathrm{~h}$. The CCK-8 assay was used to evaluate survival. The cut-off concentration, $0.0001 \%$ is presented in Fig. 4A. Ethylparaben above this level was deleterious to CFs and suppressed CF survival. However, ethylparaben below this level did no significant damage to CFs. Consequently, $0.00005 \%$ ethylparaben was recognized as eligible for exploration of the etiology of expression of ECM and agents linked with the Wnt/ $\beta$-catenin axis triggered by ethylparaben in CFs. It was demonstrated that the expression of $\mathrm{FN}-1$, Coll $\alpha 1$, collagen I, $\alpha$-SMA, total and $p-\beta$-catenin was markedly promoted $24 \mathrm{~h}$ following supplementation of CFs with $0.00005 \%$ ethylparaben (Fig. 4B). In addition, ethyparaben treatment promoted $\beta$-catenin translocation (Fig. 4C). These findings verified that ethylparaben at a low level did no damage to $\mathrm{CFs}$ and activated $\mathrm{CF}$ expression of ECM and agents linked with the Wnt/ $\beta$-catenin axis.

Ethylparaben at a low level promotes the CF expression of ECM by stimulating the Wnt/ $\beta$-catenin axis. To evaluate whether ECM gene expression was triggered by Wnt/ $/$-catenin stimulation via low levels of ethylparaben in $\mathrm{CFs}$, CFs were supplemented with ethylparaben, ethylparaben+XAV-939, or PBS. WB revealed that $\mathrm{p}-\beta$-catenin expression was markedly promoted in the experimental group compared with the control group. However, $p-\beta$-catenin concentration was markedly downregulated in CFs that received ethylparaben+XAV-939 compared with those that received ethylparaben (Fig. 5A). Similarly, FN-1, Col1 $\alpha 1$, collagen I and $\alpha$-SMA expression were markedly suppressed in CFs that
A

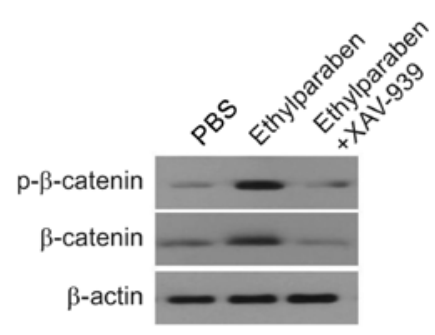

B

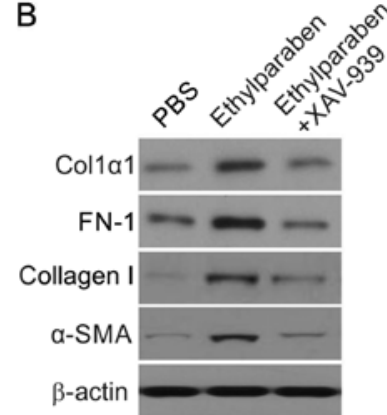

Figure 5. Effect of $\beta$-catenin inhibitor XAV-939 on the expression of p- $\beta$-catenin, Coll $\alpha 1$, and FN-1. (A) Western blot assay of expression of p- $\beta$-catenin in CFs of ethylparaben and ethylparaben+XAV-939 $(1 \mu \mathrm{M})$-treated groups. $p$ - $\beta$-catenin concentration was markedly suppressed in CFs that received ethylparaben+XAV-939 $(1 \mu \mathrm{M})$ compared with those that received ethylparaben (B) Western blotting assay of expression of FN-1, Col1 $\alpha 1$, collagen I and $\alpha$-SMA in CFs in ethylparaben and ethylparaben+XAV-939 $(1 \mu \mathrm{M})$-treated groups. FN-1, Col1 $\alpha 1$, collagen I and $\alpha$-SMA expression was markedly suppressed in CFs that received ethylparaben+XAV-939 $(1 \mu \mathrm{M})$ compared with those that received ethylparaben. P, phosphorylated; $\mathrm{CFs}$, conjunctival fibroblasts; FN, fibronectin; Col1, collagen type 1; SMA, smooth muscle actin.

received ethylparaben+XAV-939 compared with those that received ethylparaben (Fig. 5B). These findings suggested that ethylparaben at low levels triggered the expression of ECM by stimulation of the $\mathrm{Wnt} / \beta$-catenin axis in CFs.

\section{Discussion}

In the present study, it was demonstrated for the first time that ethylparaben-induced SCF was caused by the Wnt/ß-catenin signaling pathway, to the best of our knowledge.Increased density of fibroblasts and deposition of collagen in subconjunctival tissue likely accounts for $\operatorname{SCF}(28,29)$. The present study initially revealed that chronic ethylparaben supplementation mildly promoted fibroblast density and induced tighter deposition of collagen in murine BST. Chronic ethylparaben supplementation markedly promoted ECM gene expression and stimulated the Wnt//-catenin axis in murine BCT. Low levels of ethylparaben 
did no marked harm to cultivated $\mathrm{CFs}$, but promoted ECM gene expression and triggered the Wnt/ $\beta$-catenin axis. The ECM gene expression triggered via ethylparaben was impaired by the Wnt $/ \beta$-catenin inhibitor, XAV-939. These findings elucidate the etiology of SCF triggered by ethylparaben.

Ethylparaben at a low level potentially serves as a xenobiotic to promote ECM gene expression in CFs by stimulation of the Wnt/ $\beta$-catenin axis, which may be an extra side effect of ethylparaben on the conjunctiva. In investigation of ethylparaben toxicity, cell cultivation in vitro supplements animal experiments in vivo, despite the fact that cell monolayer fails to simulate the more complicated tissue structure. The cultivated cells are widely used to explore the influence of ethylparaben on the conjunctiva (30). Several objective standards are utilized to assess ethylparaben toxicity on cultivated cells such as survival, condensation of chromatin, ROS generation, and transmembrane mitochondrial potential (31-33). In order to determine the nontoxic influence of ethylparaben on CTs below the cutoff level, primary cultivated CFs were supplemented with ethylparaben for $24 \mathrm{~h}$. It was demonstrated that ethylparaben did no harm to CFs, but mildly promoted CF proliferation. Supplementation of ethylparaben at a low level in the long-term likely accounts for CFs. Animal experiments were repeated, and the same results were obtained. Future studies will focus on how ethylparaben regulates the $\mathrm{Wnt} / \beta$-catenin signaling pathway, and the protective effect of $\mathrm{Wnt} / \beta$-catenin signaling pathway inhibition on ethylparaben-induced subconjunctival fibrosis.

In summary, the present study revealed that SCF triggered by ethylparaben results from stimulation of the $\mathrm{Wnt} / \beta$-catenin axis in CFs. These findings also suggest that the development of ethylparaben-free eye drops may be necessary for patients and will also contribute to the development of novel therapeutic agents for ethylparaben-related ocular diseases.

\section{Acknowledgements}

Not applicable.

\section{Funding}

No funding was received.

\section{Availability of data and material}

All data generated or analyzed during this study are included in this published article.

\section{Authors' contributions}

FGL and XFK conceived the study and designed the experiments, XFK and HK contributed to the data collection, performed the data analysis and interpreted the results. FGL wrote the manuscript; XFK contributed to the critical revision of article. All authors read and approved the final manuscript.

\section{Ethics approval and consent to participate}

The present study was approved by the Committee on the Ethics of Animal Experiments at Zoucheng People's Hospital (Zoucheng, China).

\section{Patient consent for publication}

Not applicable.

\section{Competing interests}

The authors declare that they have no competing interests.

\section{References}

1. Final amended report on the safety assessment of Methylparaben, Ethylparaben, Propylparaben, Isopropylparaben, Butylparaben, Isobutylparaben, and Benzylparaben as used in cosmetic products. Int J Toxicol 27 (Suppl 4): S1-S82, 2008.

2. Kolatorova L, Vitku J, Hampl R, Adamcova K, Skodova T, Simkova M, Parizek A, Starka L and Duskova M: Exposure to bisphenols and parabens during pregnancy and relations to steroid changes. Environ Res 163: 115-122, 2018.

3. Huang C, Wang H, Pan J, Zhou D, Chen W, Li W, Chen Y and Liu Z: Benzalkonium chloride induces subconjunctival fibrosis through the COX-2-modulated activation of a TGF- $\beta 1 / \mathrm{Smad} 3$ signaling pathway. Invest Ophthalmol Vis Sci 55: 8111-8122, 2014.

4. Yalniz-Akkaya Z, Simsek GG, Uney GO, Burcu A and Ornek F: Effect of cauterization on the subconjunctival fibrovascular reaction in rabbit eyes. Semin Ophthalmol 30: 202-205, 2015.

5. Paula JS, Ribeiro VR, Chahud F, Cannellini R, Monteiro TC, Gomes EC, Reinach PS, Rodrigues Mde L and Silva-Cunha A: Bevacizumab-loaded polyurethane subconjunctival implants: Effects on experimental glaucoma filtration surgery. J Ocul Pharmacol Ther 29: 566-573, 2013.

6. Zhan T, Rindtorff $\mathrm{N}$ and Boutros $\mathrm{M}$ : Wnt signaling in cancer. Oncogene 36: 1461-1473, 2017.

7. Clevers H: Wnt/beta-catenin signaling in development and disease. Cell 127: 469-480, 2006.

8. Krishnamurthy N and KurzrockR: Targeting the Wnt/beta-catenin pathway in cancer: Update on effectors and inhibitors. Cancer Treat Rev 62: 50-60, 2018.

9. Zhu X, Yuan C, Tian C, Li C, Nie F, Song X, Zeng R, Wu D, Hao $\mathrm{X}$ and Li L: The plant sesquiterpene lactone parthenolide inhibits Wnt/ $\beta$-catenin signaling by blocking synthesis of the transcriptional regulators TCF4/LEF1. J Biol Chem 293: 5335-5344, 2018.

10. Feng XY, Wu XS, Wang JS, Zhang CM and Wang SL: Homeobox protein MSX-1 inhibits expression of bone morphogenetic protein 2, bone morphogenetic protein 4, and lymphoid enhancer-binding factor 1 via Wnt/ $\beta$-catenin signaling to prevent differentiation of dental mesenchymal cells during the late bell stage. Eur J Oral Sci 126: 1-12, 2018.

11. Tacchelly-Benites O, Wang Z, Yang E, Benchabane H, Tian A, Randall MP and Ahmed Y: Axin phosphorylation in both Wnt-off and Wnt-on states requires the tumor suppressor APC. PLoS Genet 14: e1007178, 2018.

12. Emerick B, Schleiniger G and Boman BM: A kinetic model to study the regulation of beta-catenin, APC, and Axin in the human colonic crypt. J Math Biol 75: 1171-1202, 2017.

13. Pecina-Slaus N, Kafka A, Vladusic T, Pecina HI and Hrascan R: AXIN1 expression and localization in meningiomas and association to changes of APC and E-cadherin. Anticancer Res 36: 4583-4594, 2016.

14. Niu C, Yin L and Aisa HA: Novel furocoumarin derivatives stimulate melanogenesis in B16 melanoma cells by up-regulation of MITF and TYR family via Akt/GSK3 $\beta / \beta$-catenin signaling pathways. Int J Mol Sci 19: E746, 2018.

15. Mbom BC, Siemers KA, Ostrowski MA, Nelson WJ and Barth AI: Nek2 phosphorylates and stabilizes $\beta$-catenin at mitotic centrosomes downstream of Plk1. Mol Biol Cell 25: 977-991, 2014.

16. Dijksterhuis JP, Baljinnyam B, Stanger K, Sercan HO, Ji Y, Andres O, Rubin JS, Hannoush RN and Schulte G: Systematic mapping of WNT-FZD protein interactions reveals functional selectivity by distinct WNT-FZD pairs. J Biol Chem 290: 6789-6798, 2015. 
17. Endo Y, Beauchamp E, Woods D, Taylor WG, Toretsky JA, Uren A and Rubin JS: Wnt-3a and Dickkopf-1 stimulate neurite outgrowth in ewing tumor cells via a Frizzled3- and c-Jun $\mathrm{N}$-terminal kinase-dependent mechanism. Mol Cell Biol 28: 2368-2379, 2008.

18. Lietman C, Wu B, Lechner S, Shinar A, Sehgal M, Rossomacha E, Datta P, Sharma A, Gandhi R, Kapoor M and Young PP: Inhibition of $\mathrm{Wnt} / \beta$-catenin signaling ameliorates osteoarthritis in a murine model of experimental osteoarthritis. JCI Insight 3: 96308, 2018

19. Jeon KI, Phipps RP, Sime PJ and Huxlin KR: Antifibrotic actions of peroxisome proliferator-activated receptor gamma ligands in corneal fibroblasts are mediated by $\beta$-catenin-regulated pathways. Am J Pathol 187: 1660-1669, 2017.

20. Kato N, Shimmura S, Kawakita T, Miyashita H, Ogawa Y, Yoshida S, Higa K, Okano $\mathrm{H}$ and Tsubota K: Beta-catenin activation and epithelial-mesenchymal transition in the pathogenesis of pterygium. Invest Ophthalmol Vis Sci 48: 1511-1517, 2007.

21. ARVO statement on registering clinical trials. Invest Ophthalmol Vis Sci 47: 1-2, 2006.

22. Tong J, Zheng X, Tan X, Fletcher R, Nikolovska-Coleska Z, Yu J and Zhang L: Mcl-1 phosphorylation without degradation mediates sensitivity to HDAC inhibitors by liberating BH3-only proteins. Cancer Res 78: 4704-4715, 2018.

23. Tong J, Wang P, Tan S, Chen D, Nikolovska-Coleska Z, Zou F, Yu J and Zhang L: Mcl-1 degradation is required for targeted therapeutics to eradicate colon cancer cells. Cancer Res 77 2512-2521, 2017.

24. Brown KD, Shah MH, Liu GS, Chan EC, Crowston JG and Peshavariya HM: Transforming growth factor $\beta 1$-induced NADPH oxidase-4 expression and fibrotic response in conjunctival fibroblasts. Invest Ophthalmol Vis Sci 58: 3011-3017, 2017.
25. Livak KJ and Schmittgen TD: Analysis of relative gene expression data using real-time quantitative PCR and the 2(-Delta Delta C(T)) method. Methods 25: 402-408, 2001.

26. Chen L, Tang RZ, Ruan J, Zhu XB and Yang Y: Up-regulation of THY1 attenuates interstitial pulmonary fibrosis and promotes lung fibroblast apoptosis during acute interstitial pneumonia by blockade of the WNT signaling pathway. Cell Cycle 4: 1-12, 2019.

27. Shao $X$ and Wei X: FOXP1 enhances fibrosis via activating Wnt $/ \beta$-catenin signaling pathway in endometriosis. Am J Transl Res 10: 3610-3618, 2018.

28. Garcia-Posadas L, Soriano-Romani L, Lopez-Garcia A and Diebold Y: An engineered human conjunctival-like tissue to study ocular surface inflammatory diseases. PLoS One 12: e0171099, 2017.

29. Ekinci M, Cagatay HH, Ceylan E, Keles S, Koban Y, Gokce G, Huseyinoğlu U, Ozcan E and Oba ME: Reduction of conjunctival fibrosis after trabeculectomy using topical $\alpha$-lipoic acid in rabbit eyes. J Glaucoma 23: 372-379, 2014

30. Takahashi N: Quantitative cytotoxicity of preservatives evaluated in cell culture with Chang's human conjunctival cells-effect of temperature on cytotoxicity. Jpn J Ophthalmol 26: 234-238, 1982.

31. Martins RC, Gmurek M, Rossi AF, Corceiro V, Costa R, Quinta-Ferreira ME, Ledakowicz S and Quinta-Ferreira RM: Application of fenton oxidation to reduce the toxicity of mixed parabens. Water Sci Technol 74: 1867-1875, 2016.

32. Liu T, Li Y, Zhao X, Zhang M and Gu W: Ethylparaben affects lifespan, fecundity, and the expression levels of ERR, EcR and YPR in drosophila melanogaster. J Insect Physiol 71: 1-7, 2014.

33. Vad NM, Shaik IH, Mehvar R and Moridani MY: Metabolic bioactivation and toxicity of ethyl 4-hydroxybenzoate in human SK-MEL-28 melanoma cells. J Pharm Sci 97: 1934-1945, 2008. 